\title{
Utilization of Contrast Echocardiography to Detect Coronary Perforation
}

\author{
Amjad abu salman ${ }^{1}$, Hilmi alnsasra ${ }^{2}$, gal tsaban ${ }^{1}$, and Sergio Kobal ${ }^{3}$ \\ ${ }^{1}$ Soroka Medical Center \\ ${ }^{2}$ Soroka University Medical Center \\ ${ }^{3}$ Soroka
}

August 14, 2021

\begin{abstract}
Coronary artery perforation (CAP) is a potentially fatal complication following percutaneous coronary intervention. The suspicion for post-procedural CAP typically arises from the development of clinical hemodynamic instability of the patient and evidence of new or worsening pericardial effusion in bedside echocardiography. Following such suspicion, emergent pericardiocentesis and immediate repeated coronary angiography should be performed to assess for coronary perforation. The use of echo-contrast material, currently used to improve echocardiographic resolution and provide real-time assessment of intracardiac blood flow evaluation, has yet been described to facilitate diagnosis of such infrequent complication without delaying invasive treatment in this setting.
\end{abstract}

\section{Utilization of Contrast Echocardiography to Detect Coronary Perforation}

Amjad Abu-Salman MD ${ }^{1}$, Hilmi Alnsasra MD ${ }^{1}$, Gal Tsaban MD MPH ${ }^{1}$, Sergio L. Kobal MD ${ }^{1}$

${ }^{1}$ Department of Cardiology, Soroka University Medical Center and Faculty of Health Sciences, Ben-Gurion University of the Negev, Beer Sheva, Israel

Word count: 414

Conflicts of Interest: None for all authors

Short title: Contrast Echocardiography of Coronary Perforation

Corresponding author:

Amjad Abu-Salman MD

Rager Av. POB 84101, Beer Sheva, Israel

Tel: $+972-52-3086664$

E-mail: amjadsalman.dr@gmail.com

\section{OBSTRACT:}

Coronary artery perforation (CAP) is a potentially fatal complication following percutaneous coronary intervention $(\mathrm{PCI})^{1}$. The suspicion for post-procedural CAP typically arises from the development of clinical hemodynamic instability of the patient and evidence of new or worsening pericardial effusion in bedside echocardiography ${ }^{1}$. Following such suspicion, emergent pericardiocentesis and immediate repeated coronary angiography should be performed to assess for coronary perforation ${ }^{2}$. The use of echo-contrast material, 
currently used to improve echocardiographic resolution and provide real-time assessment of intracardiac blood flow evaluation ${ }^{3}$, has yet been described to facilitate diagnosis of such infrequent complication without delaying invasive treatment in this setting.

\section{CASE PRESENTATION :}

In this case, a 95-year-old man with a history of essential hypertension presented with non-ST elevation myocardial infarction was referred to coronary angiography. The patient underwent coronary angiography that showed significant stenosis of the proximal left anterior descending artery (LAD) (Panel A, video 1). PCI was performed with balloon pre-dilatation followed by drug-eluting stent implantation (Panel B, video 2). Shortly after the PCI, the patient felt unwell and was found to be hypotensive. An urgent transthoracic echocardiogram (TTE) showed large pericardial effusion (PE) (Panel C, video 3). Concomitantly to the preparation for urgent pericardial drain placement (Panel D), an ultrasound enhancing agent (contrast) was injected and filled the left ventricular (LV) cavity with no evidence of contrast passing directly through the LV walls. However, some contrast appeared in the pericardial space raising suspicion of perforation of the treated LAD. (Panel E, video 4). Simultaneously, the angiogram was reviewed and raised the suspicion for dye extravasation (Panel B, video 2).

After partial drainage of the pericardial fluid and re-establishment of normal blood pressure values, a repeat angiogram was performed and demonstrated dye extravasation at the distal edge of the stent confirming coronary perforation (Panel F, video 5). The perforation area was successfully sealed by the placement of a covered stent, with immediate cessation of contrast extravasation (Panel G, video 6). Repeat TTE after the drainage showed no evidence of pericardial effusion (Panel $\mathrm{H}$ ).

\section{DISCUSSION :}

we describe a case of CAP following PCI, in which the utilization of bedside contrast echocardiography enhanced urgent diagnostic assessment and management of the patient without delaying definitive treatment. Our report may suggest that contrast echocardiography may contribute to the urgent clinical assessment of patients suspected for CAP following PCI and may improve and speed diagnostic accuracy without delaying life-saving interventions.

\section{FIGURE LEGENDS}

Panel A: Coronary angiogram illustrating proximal left descending artery stenosis (arrow).

Panel B: Coronary angiogram illustrating the final result after left descending artery stenting.

Panel C: Transthoracic echocardiography four-chamber view illustrating large pericardial effusion (arrow). LA, left atrium; LV; left ventricle; RA, right atrium; RV, right ventricle.

Panel D: Transthoracic echocardiography subcostal view illustrating intra-pericardial drainage tube (arrow). LA, left atrium; LV; left ventricle; RA, right atrium; RV, right ventricle.

Panel E: Transthoracic contrast echocardiography modified four-chamber view illustrating contrast microbubbles inside the pericardial effusion (arrow). LA left atrium; LV; left ventricle.

Panel F: Repeat coronary angiogram illustrating the left anterior descending artery with the area of coronary perforation (arrow).

Panel G: Coronary angiogram illustrating the final result after sealing the perforation area with the use of a covered stent, with no further extravasation seen.

Panel H: Transthoracic echocardiography four-chamber view showing no pericardial effusion after drainage. LA, left atrium; LV; left ventricle; RA, right atrium; RV, right ventricle.

Video1: Coronary angiogram illustrating proximal left descending artery stenosis.

Video 2: Coronary angiogram illustrating the final result after left descending artery stenting. 
Video 3: Transthoracic echocardiography four-chamber view illustrating large pericardial effusion.

Video 4: Transthoracic contrast echocardiography modified four-chamber view illustrating contrast microbubbles inside the pericardial effusion.

Video 5: Repeat coronary angiogram illustrating the left anterior descending artery with the area of coronary perforation.

Video 6: Coronary angiogram illustrating the final result after sealing the perforation area with the use of a covered stent, with no further extravasation seen.

\section{REFERENCES}

1. Lemmert ME, van Bommel RJ, Diletti R, Wilschut JM, de Jaegere PP, Zijlstra F, Daemen J, Van Mieghem NM. Clinical Characteristics and Management of Coronary Artery Perforations: A Single-Center 11-Year Experience and Practical Overview. J Am Heart Assoc[Internet]. 2017 [cited 2021 May 19];6. Available from: http://ahajournals.org

2. May A, Bhagwandeen R, Collins N. Contemporary Management of Coronary Artery Perforation. Hear Lung Circ . 2019;28:e121-e125.

3. Porter TR, Mulvagh SL, Abdelmoneim SS, Becher H, Belcik JT, Bierig M, Choy J, Gaibazzi N, Gillam LD, Janardhanan R, Kutty S, Leong-Poi H, Lindner JR, Main ML, Mathias W, Park MM, Senior R, Villanueva F. Clinical Applications of Ultrasonic Enhancing Agents in Echocardiography: 2018 American Society of Echocardiography Guidelines Update. 2018 [cited 2021 May 19];Available from: https://doi.org/10.1016/j.echo.2017.11.013

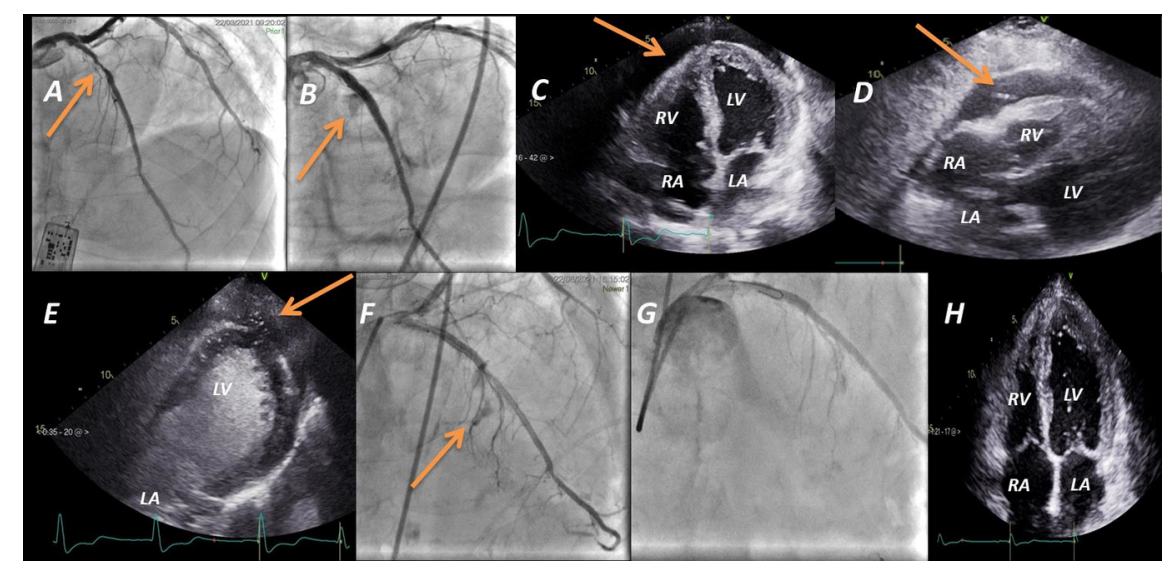

